\title{
El Frente de Liberación Nacional y Social: en busca de una definición. El PCA durante la posdictadura
}

\section{The National and Social Liberation Front: in search of a definition. The PCA during the post-dictatorship}

\author{
Natalia Casola \\ Instituto Interdisciplinario de Estudios de Género, \\ Consejo Nacional de Investigaciones Científicas y Técnicas (Argentina) \\ nataliacasola@hotmail.com
}

\section{Resumen}

El presente artículo analiza la política del Partido Comunista Argentino (PCA) durante los años 1980, es decir, en el contexto de la posdictadura y de la expectativa social generada por la recuperación de la democracia. En este trabajo se sostiene que el devenir del PCA debe leerse a la luz de una triple transición: la llamada "transición democrática" en el país, la del mundo socialista en su etapa de crisis final, y la propia, signada por una irrefrenable necesidad de cambio interno que tuvo como punto central el XVI Congreso de 1986. Toda la política partidaria desarrollada con posterioridad remonta su legitimidad a las Tesis aprobadas ese año, consideradas de un modo casi refundacional.

A grandes rasgos, el trabajo propone un análisis de la evolución de la línea partidaria, sintetizada en la estrategia de Frente de Liberación Nacional y Social (FLNS). Se trataba de una vieja consigna resignificada en el nuevo contexto. La hipótesis central de este artículo es que la línea del partido entre 1983 y 1990 fue dinámica y se caracterizó por una progresiva e ininterrumpida radicalización no exenta de indefiniciones que fueron el puntapié para las sucesivas crisis. No obstante, sistematizar los cambios en la línea, contribuirá a comprender mejor cuáles eran los debates y argumentos detrás de las rupturas.

\section{Palabras Clave}

Partido Comunista Argentino; Frente Democrático Nacional; Frente de Liberación Nacional y Social; XVI Congreso; Alianzas.

\begin{abstract}
This article analyzes the politics of the Argentine Communist Party (PCA) during the 1980s, that is, in the context of the post dictatorship and the social expectation generated by the recovery of democracy. This paper argues that the future of the PCA must be read in light of a triple transition: the so-called "democratic transition" in the country, that of the socialist world in its stage of final crisis, and its own, marked by an irrepressible need of internal change that had as its central point the XVI Congress of 1986. All the partisan politics
\end{abstract}




\section{Natalia Casola}

developed later goes back to its legitimacy to the Theses approved that year, considered in an almost refundational way.

Broadly speaking, the work proposes an analysis of the evolution of the party line, synthesized in the strategy of the National and Social Liberation Front (FLNS). It was an old resignified slogan in the new context. The central hypothesis of this article is that the party line between 1983 and 1990 was dynamic and characterized by a progressive and uninterrupted radicalization, not without its indefiniteness, which were the kick-start for the successive crises. However, systematizing the changes along the lines will contribute to a better understanding of the debates and arguments behind the ruptures.

\section{Keywords}

Argentine Communist Party; National Democratic Front; Front of National and Social Liberation; XVI Congress; Alliances.

Al finalizar la dictadura militar en 1983 la izquierda argentina debió afrontar serios desafíos. El levantamiento del Estado de excepción y el retorno de la legalidad democrática rehabilitaron un margen nuevo para la militancia pero, ideológicamente, la coyuntura se presentó hostil para los planteos de revolución. Los proyectos de inspiración marxistas constituyeron el centro de ataque tanto desde el gobierno como de los partidos políticos, las dirigencias sindicales y los medios de comunicación, quienes alentaron una interpretación sobre el pasado reciente que seguía sostenida en el consenso antisubversivo. En cierta medida, los años '80 fueron tributarios de un paradigma político organizado en torno de la antinomia "democracia vs autoritarismo" la cual dejó escaso margen para pensar la violencia revolucionaria sin quedar asociada al polo autoritario. El Nunca Más también fue sinónimo de rechazo a la violencia política. En ese contexto, los partidos de izquierda debieron abrirse un camino y hacer equilibrio entre sus programas de revolución y las presiones democráticas. El presente estudio del Partido Comunista Argentino (PCA) durante los años 1980 se inscribe en ese contexto y su devenir debe leerse a la luz de una triple transición: a. la llamada "transición democrática" en el país; b. la del mundo socialista en su etapa de crisis final; c. la propia, signada por una multicausal necesidad de cambio interno, pero que tuvo como punta de iceberg la política de "convergencia cívico-militar" sostenida durante la última dictadura. En este artículo me centraré en esta última dimensión que tuvo como punto central el XVI Congreso de 1986. Toda la política partidaria desarrollada con posterioridad remonta su legitimidad a las Tesis aprobadas ese año, consideradas de un modo casi refundacional.

En artículos anteriores he considerado la misma cuestión a la luz de la profunda crisis partidaria desatada en el segundo lustro de la década. Intenté mostrar la complejidad del proceso de revisión y cómo el aparente consenso inicial, con el "viraje" que cristalizó el XVI Congreso, se transformó en material de múltiples interpretaciones que llevaron a las sucesivas fracturas del partido en los años siguientes, en la que se mezclaron los argumentos políticos con otros de índole 


\section{EI Frente de Liberación Nacional y Social: en busca de una definición. EI PCA durante la posdictadura}

personal ${ }^{1}$. Esta política de "viraje", según sus propios términos, se proponía superar el reformismo encarnado en tres elementos entrelazados: el monolitismo burocrático, el "seguidismo" a la burguesía nacional y la política de frente "multipartidario" con los partidos tradicionales. Era el retrato de una crisis estratégica, resumida en la necesidad de dictaminar la defunción del Frente Democrático Nacional (FDN) el que, con sus contradicciones, había sido el instrumento rector de todos los armados políticos desde 1935 (Camarero, 2016). Las Tesis de 1986 cristalizaron el intento más serio por superarlo. Desde estas, se criticó la excesiva centralidad que el partido había dado a la burguesía nacional en el delineado del bloque histórico llamado a realizar la revolución democrática y, como consecuencia de lo anterior, también se intentó corregir lo que comenzó a caracterizarse como "seguidismo" al peronismo. En reemplazo del FDN se postuló la necesidad de construir un Frente de Liberación Nacional y Social (FLNS). En rigor, en el pasado, esta había sido otra forma de denominar al FDN; pero, puesto bajo el nuevo contexto, el FLNS debía representar la superación de la estrategia anterior con una definición más precisa de las fuerzas sociales y políticas llamadas a integrarlo. Entonces, ¿contra qué y quiénes se enfrentaría la revolución? ¿Qué rol desempeñarían las distintas clases y sus fracciones? ¿Cómo imaginaba el partido la dinámica interna de la revolución? ¿Cuál era el lugar asignado al propio PCA en todo ese proceso? Estas son algunas de las preguntas que permitirán comprender en qué sentido el FLNS se parecía y en qué se diferenciaba del viejo FDN o, incluso, del FLNS de antaño.

En este artículo, entonces, propongo colocar la crisis interna como telón de fondo para sistematizar la evolución de la línea principal dentro del partido como si se tratase de una sola ${ }^{2}$. Entiendo la línea como la estrategia y las tácticas decididas sobre las caracterizaciones de cada etapa y sostenidas públicamente por el conjunto del partido. Realizar el ejercicio de establecer con claridad cuáles eran las posiciones oficiales del PCA resulta un primer paso importante para comprender, luego, cómo esas posiciones se encarnaron y se materializaron (o no) en prácticas concretas.

Mi hipótesis al respecto es que el XVI Congreso buscó producir un cambio en el contenido del Frente de Liberación Nacional y Social (FLNS) al que colocó como la principal herramienta para la toma del poder y la constitución de un "gobierno popular, antiimperialista y antioligárquico" con vistas al socialismo. Si el FLNS era una consigna ya vieja que se remontaba al XI Congreso (Staltari, 2016), en la práctica, el sistema de alianzas se había movido en los marcos del Frente Democrático Nacional, que priorizaba de un modo laxo los acuerdos con el arco "progresista", en especial, desde los años 1960, con el peronismo. Fue justamente la

\footnotetext{
${ }^{1}$ Considero que la historia política no debe descuidar ni minimizar los conflictos personales. Si los vínculos de camaradería y amistad son, en ocasiones, más potentes que los manifiestos, las luchas por el poder y la acumulación de capital simbólico personal pueden desatar conflictos más agudos que de los que son capaces los enemigos.

${ }^{2}$ No es excesivo insistir, al menos en nota al pie, que en aquella coyuntura aparecieron numerosas tendencias internas, nunca reconocidas formalmente pero que tenían gravitación en los hechos. Para un primer mapeo de los alineamientos al interior del propio Comité Central, véase, Casola (2019).
} 


\section{Natalia Casola}

revisión de esos dos elementos de la línea tradicional lo que permitió redefinir el contenido del FLNS. Desde entonces, el frentismo fue imaginado como el armado para unir de forma permanente al bloque de fuerzas políticas y sociales populares de izquierda, para la toma del poder. Si bien esta propuesta implicó un cambio en la política de alianzas, introdujo nuevas indefiniciones: ¿Qué lugar cabía al PCA en ese proceso? ¿Cómo se resolvería la lucha de estrategias al interior del bloque llamado a integrar el FLNS?

Para este artículo consulté numerosos documentos y publicaciones oficiales. Sin embargo, decidí recortar el análisis en los documentos congresales e informes del Comité Central porque constituyen las instancias más formales donde rastrear la línea del partido.

Este trabajo contribuye con un campo de investigación en desarrollo que tiene como objetivo conocer la historia del PCA en los años 1980. Son varios los y las investigadoras que en la actualidad se encuentran reflexionando sobre este periodo (Bona, 2019; Ermosi, 2017; Messina, 2020); indagaciones que se suman a otras ya concluidas, pero también de reciente publicación (Fernández Hellmund, 2015; Casola, 2016; 2019). Junto con estos trabajos cabría incorporar los producidos por dirigentes o ex dirigentes del partido, los cuales constituyen una expresión testimonial del proceso analizado (Echegaray, 1996; Fava, 2006; Sigal, 2006; Schulman, 2008; Gilbert, 2010; Nadra, 2012). Estas memorias personales y reconstrucciones históricas tienen la ventaja de ofrecer el punto de vista personal e informar sobre cuestiones no fácilmente hallables en los documentos oficiales. Mirados como un conjunto, todos estos trabajos permiten establecer un piso de conocimiento sobre el PCA en el pasado reciente y dejar sentadas las bases para ampliar las agendas de investigación e incorporar nuevas preguntas y perspectivas.

\section{La política del PCA entre 1983 y 1986}

Para finales de 1983 el PCA era un partido que comenzaba a recuperar su activo militante luego del retroceso que sufrieron todas las fuerzas de izquierda durante la dictadura. Ese crecimiento se manifestaba en un estado de ánimo de la militancia que se mantuvo alto en los años siguientes, en la búsqueda de renovación y en la formulación esperanzada de la crítica (Bona, 2019)³. A pesar de la política de

\footnotetext{
${ }^{3}$ Contabilizar la militancia comunista en este periodo no es una tarea sencilla y toda afirmación al respecto debiera tomarse como provisoria. Los documentos oficiales, informes congresales y de organización, solían informar solo las cifras de afiliaciones, un dato que puede ser útil para pensar la influencia del partido, pero que no reflejaba al activo real de militantes que eran los verdaderos constructores de la organización. De todos modos, el número de militantes debió estar lejos de la cifra de 300.000 afiliados que afirmaban tener en 1983 sumando a la FJC. Un indicador de esto, por ejemplo, es la prensa partidaria que en el mejor momento de ese mismo año alcanzó la excepcional tirada de 110.000 ejemplares, un tercio de la totalidad de afiliados. Pero, además, las cifras de afiliaciones no parecieran constituir un indicador confiable por razones de contexto: ¿Cómo habían pasado de los casi 100000 afiliados de la década de1970 a los 300000 en 1983? ¿Acaso podía tomarse la campaña de afiliación en el marco de las elecciones como una adhesión automática al PCA? Otra posibilidad para efectuar un cálculo es tomar la cantidad de delegados al congreso, la cual, según los estatutos, se deciden por cantidad de afiliados. Sin embargo, no existía una cuenta estable para
} 


\section{EI Frente de Liberación Nacional y Social: en busca de una definición. EI PCA durante la posdictadura}

"convergencia cívico militar", sostenida durante la dictadura, el PCA había sabido conservar su aparato y estructura, un rasgo que lo transformó en un espacio de reagrupamiento para un sector de la población que continuaba identificándose con la izquierda, pero que había quedado huérfana de organización, o que miraba las experiencias de los años 1970 con cierto tono crítico. El programa del PCA de 1983 parecía interpretar los nuevos tiempos a la perfección. Contenía reivindicaciones de izquierda enmarcadas en una estrategia que difícilmente podría clasificarse como revolucionaria ya que seguía encuadrada en los marcos del FDN, buscaba consensos con los partidos tradicionales (en especial el PJ y la UCR) y su reivindicación de la democracia se enunciaba en términos tan generales que parecía despojada de su contenido de clase. Es decir que el nivel de conflictividad que proponía el PCA en 1983 era bajo. Pese a eso, o justamente por eso, en un contexto de fuerte descrédito de los paradigmas revolucionarios y de revalorización de la democracia liberal, ese capital político podía ser capitalizado.

Desde el punto de vista cupular, la dirigencia del PCA era prácticamente la misma que había llevado las riendas del partido desde el último congreso realizado en 1973. En 1983 se realizó el XV Congreso que ratificó la continuidad de la mayoría de los miembros del Comité Central y su Secretariado Nacional, con excepción de unos pocos cambios producto del fallecimiento de algunos antiguos dirigentes. Puede pensarse que esa estabilidad en las planas dirigenciales se reflejó en la continuidad de los marcos interpretativos que daban contenido a la línea.

Durante estos años, los ejes de la política partidaria giraron en torno a dos problemas: 1. la cuestión de la crisis económica y la propuesta de "moratoria" para la deuda externa; y 2 . la defensa de la democracia.

Con respecto al primer problema, la política del partido confrontaba con los planes de gobierno y denunciaba sus efectos sobre los trabajadores y sobre la estructura productiva del país. En relación con el segundo problema el PCA participaba de los extendidos temores sociales acerca de la posibilidad de un nuevo golpe de Estado. Por eso, el XV Congreso se expresó en favor de luchar "por la estabilidad del nuevo gobierno" (Fava, 1983: 18). A la luz de la fragilidad histórica de la democracia argentina y de situaciones puntuales que buscaban crear terror y una sensación de inestabilidad política y debilidad gubernamental — que el propio alfonsinismo supo explotar a su tiempo-, no se trataba de un temor injustificado.

Esa caracterización inicial condicionó la política frentista imaginada por el partido. Durante 1983 y 1984 la misma siguió en los cauces abiertos durante la dictadura cuando el PCA celebró la constitución de la Multipartidaria. Esa política había

establecer cada cuántos militantes se elegía un delegado lo que constituía una atribución del Comité Central convocante. La cantidad de delegados, por si fuera poco, era muy fluctuante. Por caso el XIV Congreso contó con 507 delegados, del XV participaron 176, mientras que en el XVI participaron 673 delegados. Los datos difundidos sobre el XVI Congreso afirmaban haber elegido sus 673 delegados en 2000 asambleas de organismos básicos. Si nuevamente tomamos el número de 10 militantes por célula nos da un total de 20000 militantes, quizás la cifra más realista que hasta el momento hemos barajado. 
llevado al partido, en 1983, al extremo de declinar su propia candidatura presidencial para apoyar al candidato peronista Italo Lúder. El XV Congreso cristalizó esta apuesta en el llamado "perocomunismo".

Si bien el voto a Lúder y a Herminio Iglesias no tardó en ser motivo de crítica interna - porque se trataba de dos personajes identificados claramente con la derecha del Justicialismo-, esto que no alcanzó para romper decididamente con la aspiración a construir un espacio de convergencia con el PJ. La cuestión del peronismo había sido central para el PCA desde los años 1940, pero mucho más desde que Codovilla decretara el "giro a la izquierda" en 1962. Sin embargo, si la política del giro a la izquierda había sido pensada, al menos en teoría, para traccionar a los trabajadores peronistas hacia la izquierda, en la práctica, el movimiento había sido el contrario. Todavía en 1985, Athos Fava, afirmaba que confiaba en el "giro a la izquierda del peronismo". Reproduzco en extenso una parte de su informe dirigido al Comité Central porque considero que es esclarecedor de la visión que se tenía entonces:

\begin{abstract}
“[...] está la crisis del peronismo, más aguda que nunca, a la que nos referiremos más adelante, pero en cuyo trasfondo hay un desarrollo: el giro a la izquierda [...]. El giro a la izquierda y la conciencia antiimperialista es lo que se abre paso en las masas peronistas. Este giro analizado por Victorio Codovilla en 1962, hoy es más definido. Y cuando hablamos de los cambios en el peronismo no nos referimos sólo a su base obrera y popular, sino también a sus dirigentes en TODOS sus niveles. No verlo así es también un rasgo de sectarismo que debemos combatir. El estrechamiento de lazos, la unidad en la lucha, los acuerdos y las acciones con esas masas peronistas, principalmente en el movimiento obrero, han sido y seguirán siendo el centro de nuestros desvelos. [...] Por eso cabe un interrogante: $i S e$ mantendrá el peronismo en los viejos moldes policlasistas y conciliadores de heterogeneidad [...]; o por el contrario se abrirá paso una corriente peronista que recoja las banderas liberadoras, antioligárquicas y antiimperialistas del propio justicialista? Todo indica que esta última es la tendencia general" (Fava, 1985: 9 y10).
\end{abstract}

Los pasajes seleccionados son suficientemente representativos de la pervivencia de una concepción que aseguraba que el peronismo podía ser traccionado hacia las posiciones del partido. Pero si en las décadas de 1960 y 1970 esa orientación podía apoyarse en la creciente actividad del peronismo de izquierda y en la recurrencia de regímenes militares que perseguían tanto al comunismo como a los peronistas, en 1985 la situación era muy diferente. Se omitía que los sectores del peronismo combativo eran justamente los que habían quedado más desarticulados por la política de exterminio de la dictadura. El peronismo que emergía en 1983 seguía siendo heterogéneo, pero en su conjunto había declinado de los horizontes de "socialismo nacional".

La política de alianzas del PCA, entonces, seguía en los carriles de los acuerdos multipartidarios, buscando converger con un arco amplio identificado de un modo general con el progresismo y la democracia. El partido sostenía expectativas en el peronismo, pero también en algunos sectores de la UCR. Por esa razón, todavía en 1983 proponía apuntalar la Multipartidaria “y su plataforma común de la 


\title{
EI Frente de Liberación Nacional y Social: en busca de una definición. EI PCA durante la posdictadura
}

democracia"4. En consecuencia, prevalecía en estos años una visión frentista adecuada con la línea histórica del FDN. En 1983 el planteo era similar al que habían sostenido en 1973 y apuntaba a la unidad de las fuerzas democráticas.

\begin{abstract}
"Recogiendo esta experiencia que hicimos juntos todos los sectores patrióticos y democráticos del pueblo, debemos desterrar todo lo que trabe la tendencia a la unidad [...]. Solo así podrá abrirse paso a una nueva experiencia que nos lleve a cerrar el ciclo nefasto de los golpes de Estado abierto en 1930 y a evitar el peligro de la disolución nacional y la guerra civil. Para ello es imprescindible llegar a un convenio, o a una carta democrática, o a un pacto de garantías constitucionales o como quiera llamársele: al necesario acuerdo para lograr la estabilidad democrática y verdadera" (Fava, 1983:31).
\end{abstract}

Como puede verse, eran las antinomias "democracia vs. dictadura"; "progreso vs. reacción", las que aún organizaban las elecciones frentistas del PCA.

Un año después, y al calor de las críticas que comenzaban a circular por lo bajo con relación a la política sostenida en dictadura y durante la campaña electoral, un sector pequeño de la dirigencia comunista comenzó a recuperar la consigna de FLNS5. La lectura sobre los acontecimientos en Nicaragua fue clave porque proporcionó un ejemplo de revolución triunfante en la que confluían diferentes fuerzas sociales y políticas aunadas en post de un objetivo mayor: derrocar a Somoza.

¿Qué diferenciaba al FLNS del FDN? Ambas denominaciones proponían la necesidad de construir alianzas con todos los sectores sociales y políticos interesados en luchar contra el imperialismo, contra el capital monopólico y contra la oligarquía. ¿Qué lugar cabía a la burguesía nacional, mediana o pequeña, del campo y la ciudad, en ese frente? ¿Cuáles eran los partidos políticos y organizaciones que compartían con el comunismo esa lucha? Para el comunismo del primer lustro de los años 1980 no había muchas dudas: la burguesía nacional y la pequeña burguesía todavía podían transformarse en sujetos de lucha contra el capital concentrado, bajo la presión y dirección de los trabajadores organizados (y de allí la necesidad de la existencia de un partido comunista cuya función debía ser garantizar esa hegemonía). Con relación a las fuerzas políticas que integrarían el frente aún eran muy variadas. Una imagen que resumen bien la concepción de frente que imaginaba el PCA fue la que ilustró la tapa de la revista Nueva Era, en su número de diciembre de 1985. En ella se ven puños levantados, manos haciendo la $\mathrm{V}$ (signo inequívoco de peronismo) y el puño y la rosa, en representación del socialismo, todas juntas y aunadas por una especie de aura que las mancomunaba.

\footnotetext{
5 De acuerdo con las memorias de Alberto Nadra, este grupo, del que formaba parte, estaba conformado, además, por Patricio Echegaray, Francisco “Cacho” Álvarez y Enrique Dratman. Siempre siguiendo este testimonio, habría sido Echegaray el autor de la alianza estratégica con Athos Fava y Jorge Pereyra que permitió formular el "viraje en unidad" y abrir un camino para los planteos de renovación al interior del Comité Central, controlado aún por dirigentes que comenzaron a ser denominados como "dinosaurios" (Nadra, 2012: 107).
} 


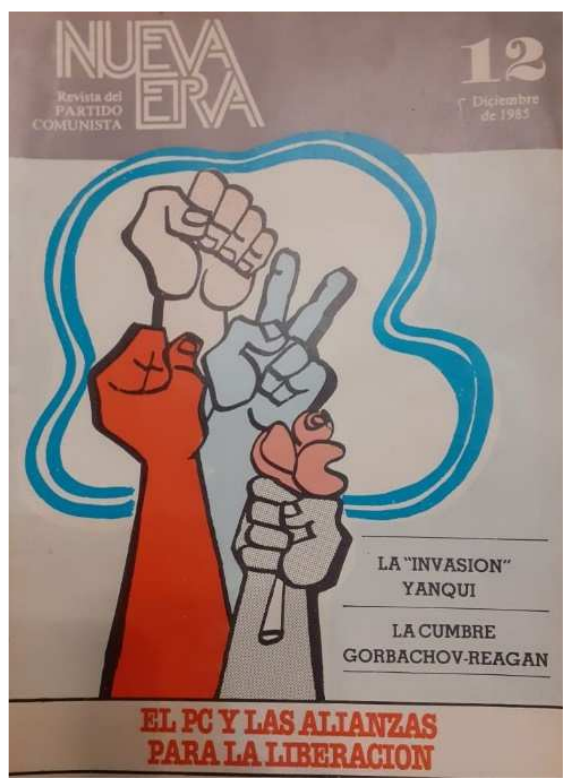

Tapa de la revista Nueva Era, n²12, diciembre de 1985.

Fuente: Archivo personal de la autora

En esta primera etapa, por tanto, se observan rasgos de continuidad con la línea del FDN, entendido como frente amplio para la defensa de un programa de revolución democrática, antioligárquica y antiimperialista. El primer elemento disruptivo fue la constitución del Frente del Pueblo (FREPU) con el Movimiento al Socialismo (MAS) con vistas a las elecciones legislativas de noviembre de 1985. En la práctica, se trató de una experiencia corta que no superó el mero acuerdo electoral y que generó tensiones al interior del partido. Sin embargo, no era usual que los documentos oficiales previos al XVI Congreso hicieran referencias concretas a los desacuerdos internos y, cuando estos se admitían, generalmente eran minimizados. No obstante, en un documento firmado por Athos Fava se afirmaba que "hay quienes piensan entre nosotros, los comunistas, que la alianza con el MAS crea dificultades adicionales a las que ya existen para profundizar la relación entre comunistas y peronistas en el movimiento obrero". Como puede verse, la alianza con el trotskismo generaba incomodidad en algunos sectores del partido. Resulta difícil comprender de manera acabada por qué existía resistencia a estrechar lazos con la izquierda si no se atiende suficientemente a factores relacionados con la cultura partidaria. En efecto, durante décadas la militancia comunista de todo el mundo había sido educada en la lucha contra el trotskismo y el partido argentino no era la excepción. El PCA solía auto representarse como un partido no sectario y flexible para establecer acuerdos. Sin embargo, esa apertura pocas veces se había promovido hacia la izquierda marxista, que en el pasado había sido peyorativamente caracterizada como "ultra" e incapaz de comprender el peronismo.

En esta etapa, ya comenzaban a explicitarse las críticas a la política del partido, a sus posiciones durante la dictadura, a su reformismo, a la política electoral de 1983. A 


\section{EI Frente de Liberación Nacional y Social: en busca de una definición. EI PCA durante la posdictadura}

esto se sumaba el burocratismo de su dirigencia, aunque aún prevalecía en la letra escrita del discurso oficial una defensa general de lo actuado en los últimos años, incluyendo la política de "convergencia cívico militar". En un informe rendido al Comité Central, Jorge Pereyra, el poderoso Secretario de Organización y quien luego se convertiría en patrocinador del grupo revisionista, afirmaba que el partido había logrado mantener gran parte de su activo en dictadura gracias a la justeza de su línea y de las tácticas empleadas (Pereyra, 1984:18).

Un hecho bisagra que terminaría de fijar el rumbo del viraje y el enfrentamiento al interior del partido ocurrió en 1985, cuando el PCA acudió a la Plaza de Mayo respondiendo al llamado de Alfonsín a "defender la democracia", escenario que el presidente utilizó como plataforma para el lanzamiento de la "economía de guerra". En ese contexto, el sector que impulsaba el viraje promovió el retiro del PCA de la plaza.

\section{La política del PCA entre 1986 y 1990}

Desde el punto de vista de la conducción partidaria, el XVI Congreso plasmó cambios importantes. En primer lugar, el número de integrantes del Comité Central pasó de 92 a 100. Sin embargo, ese crecimiento que podría interpretarse como la expresión de una mayor diversidad y ampliación de las voces, se compensó con una disminución del número que integraba el Secretariado, que pasó de 17 a 12. Es decir que el control del viraje se garantizó con una mayor centralización del poder a contrapelo de lo que cabría suponer con un Comité Central tan amplio.

Para terminar de comprender hasta qué punto el Comité Central había cambiado es necesario ver el flujo en la rotación de los cargos entre un congreso y el siguiente. Si se tiene en cuenta la estabilidad que había caracterizado históricamente a la dirigencia comunista, dado que buena parte de los egresos solía producirse por fallecimientos, el nivel de rotación del XVI Congreso fue muy alto. Desde luego, no se trató de una camada nueva en el sentido estricto del término. La totalidad de los cuadros elegidos tenía larga experiencia dentro del partido y, a su modo, habían participado de todos los "errores" que se buscaba corregir. Sin embargo, es cierto que, salvo algunas excepciones, para la mayoría de los ingresantes la llegada al Comité Central plasmó un proceso de ascenso rápido en el funcionariado partidario. Como hemos fundamentado en anteriores trabajos, este cambio fue precedido por la promoción de numerosos dirigentes de la FJC al partido, lo que modificó la composición del Congreso en 1986. De acuerdo con el Estatuto que regulaba la vida interna del partido, la FJC estaba representada en esta instancia por "una delegación única, equivalente en número a la más numerosa de las delegaciones partidarias" (Estatuto del Partido Comunista de la Argentina, 1974). De este modo, la promoción de dirigentes previa al Congreso pudo haber incidido en su composición en favor del núcleo renovador. 


\begin{tabular}{|c|c|c|}
\hline $\begin{array}{l}\text { MIENTROS DEL COMITÉ } \\
\text { EJECUTIVO } 1974\end{array}$ & $\begin{array}{l}\text { MIENBROS DEL COMITÉ } \\
\text { EJECUTIVO } 1983\end{array}$ & $\begin{array}{l}\text { MIEMBROS DEL COMITÉ } \\
\text { EJECUTIVO } 1986\end{array}$ \\
\hline Agosti Héctor & Agosti Héctor & XXXXXXX Fallecido \\
\hline Arévalo Oscar & Arévalo Oscar & XXXXXXX \\
\hline De La Peña Alcira & De La Peña Alcira & $\begin{array}{l}\text { xxxxxхxхxхxx Es parte de la } \\
\text { presidencia del Congreso. }\end{array}$ \\
\hline Fava Athos & Fava Athos & Fava Athos \\
\hline García José & Xxxxxxxxxx & xxxxxxxxxxxxxxxxxx \\
\hline Gerónimo Arnedo Alvarez & xxxxxxxxxx muere en 1980 & xxxxxxxxxxxxxxxxxx \\
\hline Ghioldi Orestes & Xxxxxxxxxxx & xxxxxxxxxxxxxxxxxx \\
\hline Ghioldi Rodolfo & Ghioldi Rodolfo & xxxxxxxxxxxxxxxxxx \\
\hline Iscaro Rubens & Iscaro Rubens & xxxxxxxxxxxxxxxxxx \\
\hline Rodríguez Irene & Rodríguez Irene & $\begin{array}{l}\text { xxxxxxxxxxxxxxxxxx Continúa } \\
\text { en el CC }\end{array}$ \\
\hline Mariani Ariel & $\begin{array}{l}\text { xxxxxxxxxxxxxx pasa a } \\
\text { suplente }\end{array}$ & xxxxxxxxxxxxxxxxxx \\
\hline $\begin{array}{l}\text { Moretti Florindo muere en } \\
1984\end{array}$ & Xxxxxxxxxxxxxx & xxxxxxxxxxxxxxxxxx \\
\hline Nadra Fernando & Nadra Fernando & $\begin{array}{l}\text { xxxxxxxxxxxxxxxxxx Continúa } \\
\text { en el CC y también Alberto }\end{array}$ \\
\hline Ojeda Hugo & Ojeda Hugo & xxxxxxxxxxxxxxxxxx \\
\hline Pereyra Jorge & Pereyra Jorge & Jorge Pereyra \\
\hline Santaren Héctor & Xxxxxxxxxxxxxx & $\begin{array}{l}\text { xxxxxxxxxxxxxxxxxx Continúa } \\
\text { en el CC }\end{array}$ \\
\hline Tadioli Pedro & Tadioli Pedro & xxxxxxxxxxxxxxxxxx \\
\hline XXXXXXXXXXXXXXx & Martinovich Antonio & xxxxxxxxxxxxxxxxxx \\
\hline XXXXXXXXXXXXXXX & Occhipinti Juan & xxxxxxxxxxxxxxxxxx \\
\hline Xxxxxxxxxxxxxxx & Clementi Ricardo & xxxxxxxxxxxxxxxxxx \\
\hline XXXXXXXXXXXXXXX & Heller Luis & Heller Luis \\
\hline Xxxxxxxxxxxxxxx & Echegaray Patricio (FJC) & Echegaray Patricio \\
\hline \multirow[t]{8}{*}{ Xxxxxxxxxxxxxxx } & Edelman Fanny & Edelman Fanny \\
\hline & & Ernesto Salgado \\
\hline & & Ballato Miguel \\
\hline & & Varone Guillermo \\
\hline & & Sigal Eduardo \\
\hline & & Casals Rodolfo \\
\hline & & Dratman Enrique \\
\hline & & Alvarez Francisco \\
\hline Total 17 miembros & Total 17 miembros & Total 12 miembros \\
\hline
\end{tabular}

Cuadro 1: Evolución en la composición del Comité Ejecutivo del PCA entre 1974 y 1986.

Fuente: elaboración propia. 
El Frente de Liberación Nacional y Social: en busca de una definición. El PCA durante la posdictadura

\begin{tabular}{|l|l|l|}
\hline MIEMBROS DEL & MIEMBROS DEL & MIEMBROS \\
SECRETARIADO & SECRETARIADO DEL \\
NACIONAL 1974 & NACIONAL 1983 & $\mathbf{1 9 8 6}$ \\
\hline Pereyra Jorge & Pereyra Jorge & Pereyra Jorge \\
\hline xxxxxxxxxxx & Arévalo Oscar & Xxxxxxxxxxxxx \\
\hline xxxxxxxxxxx & Nadra Fernando & Xxxxxxxxxxxxx \\
\hline Iscaro Rubens & Iscaro Rubens & Xxxxxxxxxxxxx \\
\hline Rodríguez Irene & Rodríguez Irene & xxxxxxxxxxxxx Pasa al CC \\
\hline Arnedo Alvarez Gerónimo & Xxxxxxxxxxxxxxxxxxx & Xxxxxxxxxxxxx \\
\hline Fava Athos & Fava Athos (Pasa a & Fava Athos \\
& Secretario General) & \\
\hline Ghioldi Orestes & xxxxxxxxxxxxxxxxxxx & Xxxxxxxxxxxxx \\
\hline Tadioli Pedro & xxxxxxxxxxxxxxxxxx & Xxxxxxxxxxxxx \\
\hline & & Echegaray Patricio \\
\hline & & Salgado Ernesto \\
\hline & & Heller Luis \\
\hline
\end{tabular}

Cuadro 2: Evolución del Secretariado Nacional entre 1974 y 1986. Fuente: elaboración propia.

En suma, el análisis de la dirigencia comunista nos muestra que los cambios en la línea política acompañaron la renovación en los cuadros ejecutivos. Rotación y centralización del control en un Secretariado más pequeño parecen haber sido los dos movimientos cupulares para garantizar los cambios en la línea y mantener a raya el surgimiento de las disidencias.

Desde el punto de vista programático, a partir del XVI Congreso la política oficial decantó en el sentido propuesto por los grupos renovadores y se profundizaron los rasgos más radicales que venían insinuándose en el período previo. El FLNS fue transformado en el corazón de la estrategia partidaria y adquirió un contenido más específico que el que había tenido en el pasado. Pasó a concebirse como el instrumento principal para "la toma del poder" en el marco de un proyecto de "liberación nacional y social". Entre los componentes novedosos de esta formulación se destacó el reposicionamiento de la clase obrera como sujeto principal y director del proyecto revolucionario. Vale aclarar que en las Tesis congresales se empleaba la categoría "clase obrera" en un sentido restringido y, por esa razón, se prescribía la necesidad de ampliar el universo de fuerzas sociales al "pueblo", concepto que incluía a todos los asalariados y a los sectores de la pequeña burguesía de la ciudad y del campo, cuyos intereses entraban en contradicción con el imperialismo y el capital monopolista. Si bien las históricas formulaciones frentepopulistas no desaparecían por completo, bajo el paraguas del "pueblo" asomaba la voluntad de imponer una tesis novedosa, no para el marxismo, pero sí para el PCA: se afirmaba que la burguesía nacional era incapaz de llevar a cabo como clase un proyecto de desarrollo independiente en una Argentina cuya dependencia del imperialismo se había reforzado. Esas transformaciones estructurales incapacitaban por completo a 


\section{Natalia Casola}

la burguesía nacional en su misión de desarrollar un modelo económico independiente.

Otra cuestión importante es que el FLNS comenzó a ser pensado como una herramienta al servicio de la unidad de la izquierda. Es decir, que aparecía explicitada la ruptura respecto del Frente Democrático Nacional, multipartidario, tal y como había sido concebido por décadas. Como se verá a continuación, se trataba de una novedad importante pero que entrañaba algunas imprecisiones. En primer lugar, porque desconsideraba la fuerte lucha ideológica al interior del campo de la izquierda, una cuestión que no se reducía al supuesto "sectarismo congénito" de la enorme familia marxista, sino que tenía que ver con la contraposición de estrategias diferentes. Segundo, porque la amplitud del FLNS buscaba incorporar a todos los sectores antiimperialistas, un registro que podía ser perfectamente compartido por muchas fuerzas políticas del nacionalismo popular sin llegar a plantear por eso la necesidad de hacer la revolución en un sentido socialista.

A pesar de los conflictos o tensiones que dejaba planteada la propuesta, el conjunto de las tesis suponía revisar la caracterización sobre varias cuestiones: el papel de los partidos tradicionales (la UCR y el PJ), el tipo de régimen político y el rol del imperialismo estadounidense en ese contexto.

Si entre 1983 y 1985 los materiales del partido todavía colocaban expectativas en la democracia y su capacidad para transformar la realidad, a partir del XVI Congreso la caracterización comenzó a mostrar cambios. El PCA consideraba que en la Argentina se había impuesto un modelo de democracia restringida, expresada en el bipartidismo con tutela militar. De acuerdo con esta nueva lectura, el imperialismo apuntaba a los conflictos de baja intensidad. Se trataba de un cambio importante porque se reconocía que el imperialismo norteamericano estaba interesado en la estabilización de la democracia y, a diferencia de lo que había ocurrido en el pasado, la utilizaba como puntal de la contrarrevolución. Esto no quiere decir que el PCA no reconociera en los planteos militares un factor de posible desestabilización política, sino que no consideraban que estos lograran apoyos mayoritarios. Su caracterización de la violencia estatal en democracia se había vuelto más precisa:

\footnotetext{
"Se trata en los marcos del conflicto de baja intensidad de incorporar la violencia organizada de clase como una variable normal de la lucha política coexistiendo con un sistema de representación política 'democrático'. Su objetivo es aislar a través del terror, a la militancia capaz potencialmente de orientar y dirigir las luchas de forma tal de generar un serio escollo con el proceso de acumulación político y organizativo en el seno del campo popular. [...] En esta etapa fueron asesinados los compañeros "chingolito" Villanueva y Lezcano" (Informe del Comité Central, 1987:12).
}

Con esta afirmación el PCA avanzaba en una nueva caracterización sobre el significado de la represión. Así, la represión dejaba de verse como un resabio de la dictadura o como la acción de bandas de derecha con vistas a desestabilizar la democracia, para integrarla en una lectura que reforzaba el concepto de democracia restringida, basada en la "modernización de la dependencia" (el neoliberalismo) e 


\section{EI Frente de Liberación Nacional y Social: en busca de una definición. EI PCA durante la posdictadura}

incompatible con una democracia con justicia social. La democracia restringida a lo procedimental contenía necesarias dosis de represión para disciplinar las luchas obreras y a los sectores opositores. Esta caracterización encuadraba dentro un enfoque más general organizado a partir de la antinomia "liberación o dependencia". En tanto, la democracia ampliada, real y participativa solo era posible en los marcos de un proyecto nacional enfrentado al imperialismo y a la gran burguesía nacional asociada a aquel.

En consecuencia, el PCA comenzó a delimitarse con mayor definición con respecto a la UCR y al peronismo renovador, dos expresiones que, en la visión post viraje, eran coincidentes en lo esencial del programa sostenido por el capital monopolista y el imperialismo: privatizaciones, regimentación obrera, impunidad para los aparatos represivos, etc. No obstante, la caracterización oficial respecto del peronismo, ganó en precisión: se siguió apelando a la necesidad de discutir con los militantes de base pero cuidando la delimitación respecto de la dirigencia.

En esta etapa el FLNS adquirió un contenido de izquierda más definido. El primer ensayo había sido el FP, que se presentó a elecciones en 1985. La experiencia duró poco tiempo y los motivos de disolución preanunciaban un debate que seguiría latente en los años posteriores: mientras que para el MAS el FP debía ser la expresión de un "frente de los trabajadores", es decir una herramienta de independencia de clase, para el PCA, en cambio, el FP debía expresar un "frente de liberación nacional y social", una formulación más laxa que admitía la incorporación de sectores políticos no identificados con el anticapitalismo. Pese a las diferencias, en la visión del PCA la apuesta política había sido correcta. ${ }^{6}$ El 1 de mayo de 1987, luego de un acto en el estadio del club Atlanta de la Ciudad de Buenos Aires, se lanzó, con la consigna de "El Frente Va", una nueva apuesta: el Frente Amplio Latinoamericano (FRAL). Este nuevo armado político era concebido como un instrumento para la unidad de la izquierda y estaba compuesto por varias organizaciones pequeñas, entre las que destacaba, además del propio PCA, el Partido Humanista (PH), Patria Libre, la 29 de Mayo y la 26 de Julio. En septiembre de ese año las elecciones arrojaron resultados magros para la coalición, incluso por detrás de los pronósticos partidarios. Sin embargo, en los balances posteriores se evaluó de manera positiva la acción política y se instó a profundizar en la unidad de la izquierda, lo que implicaba retomar las discusiones con el MAS. No obstante, para el PCA, la izquierda seguía sin legitimarse como alternativa de cambio y esa era la razón por la que las masas habían decidido castigar al radicalismo volcando su voto en el peronismo renovador. Simultáneamente, la caracterización del peronismo perdía la ambigüedad que había tenido en el pasado y se afirmaba que:

[...] "en la dirección del Partido Justicialista se ha consolidado la influencia de la burguesía monopolista, como fracción hegemónica, secundada por personeros del viejo peronismo y la burocracia sindical. [...] Se trata, por otra parte, de un

\footnotetext{
${ }^{6}$ No obstante, el PCA no pudo evitar la ruptura del FP la cual se produjo por divergencias con relación a la sanción de las leyes de Punto Final y Obediencia Debida.
} 


\title{
Natalia Casola
}

partido del sistema. Eso está claro, Pero la base, a diferencia del radicalismo, está constituida por la clase obrera y los sectores más humildes, cuyos reclamos pueden llegar a perturbar el programa de modernización" (Informe del Comité Central, 1987:14).

Para finales de 1987, el informe del Comité Central afirmaba que existía acuerdo entre el radicalismo, los renovadores y la UCEDE para afianzar el modelo de apertura, privatización, desregulación, atracción de capitales y capitalización de la deuda. Dicho informe, además, sostenía que la crisis se profundizaría y que tanto las cúpulas de la UCR como la del PJ se verían obligadas a desempolvar propuestas demagógicas en vistas a las elecciones de 1989.

"Por lo tanto, no es posible hacerse ilusiones de que el peronismo pueda, en los marcos del sistema que propone, presentar una alternativa diferente, un tercer camino de desarrollo del capitalismo" (Informe del Comité Central, 1987: 4).

Como se ha mencionado en trabajos anteriores, esta nueva orientación fue problemática hacia el interior del partido y llevó a que el propio CC reconociera una disminución del activismo acostumbrado y educado en la construcción conjunta con el peronismo, especialmente en los sindicatos.

Además, en estos años el crecimiento del PCA estuvo condicionado por el crecimiento del MAS que también había atravesado un proceso revisionista. En términos electorales, los comicios de 1987 mostraron hasta qué punto el MAS había ganado gravitación: en los distritos más poblados del país obtuvo casi la misma cantidad de votos que el FRAL. Para el PCA esto significaba que debía restablecerse el diálogo con el MAS e intentar traccionarlo hacia su espacio.

\begin{abstract}
"Tenemos que trabajar para que otros destacamentos de la izquierda adopten la estrategia del FLNS, de la unidad de la izquierda, del aglutinamiento de la fuerza social y política de la revolución. [...] Reafirmar la concepción del FLNS como el instrumento político de las masas para conformar el bloque popular capaz de conquistar el poder político, entendido como un sistema de alianzas políticas y sociales" (Informe del Comité Central, 1987: 28).
\end{abstract}

Durante 1988, el PCA, junto con otras organizaciones aliadas, puso a prueba la estrategia de FLNS: la movilización a los cuarteles y el enfrentamiento con las fracciones militares en Monte Caseros y Villa Martelli. Con esta acción en común se intentó pasar del acuerdo electoral a la política de unidad en la acción.

Sin embargo, el inicio de 1989 estuvo atravesado por una enorme conflictividad que puso en el banquillo a toda la izquierda. El 23 de enero se produjo el intento fraguado de copamiento, por parte del Movimiento Todos por la Patria (MTP)7 , del Tercer Regimiento de Infantería Mecanizada General Belgrano (RIM 3) ubicado en

\footnotetext{
${ }^{7}$ El enfrentamiento, que duró 27 horas, dejó un saldo de 43 muertos y decenas de heridos. El MTP había surgido en el fragor de la lucha en Nicaragua y era dirigido por Gorriarán Merlo, quien anteriormente había militado en el Partido Revolucionario de los Trabajadores (PRT).
} 


\section{EI Frente de Liberación Nacional y Social: en busca de una definición. EI PCA durante la posdictadura}

la localidad de La Tablada. El MTP había surgido en 1985 y agrupaba, especialmente, a ex militantes del PRT. Si bien sostenía planteos parecidos a los del PCA, el MTP consideraba que los levantamientos carapintadas constituían un intento serio de golpe de Estado. Y aunque existían vasos comunicantes entre algunos sectores del PCA con el MTP, la postura oficial y pública en aquellos días fue delimitarse con claridad. De todos modos, y a pesar de polemizar con el contenido de la acción, el partido ofreció ayuda jurídica a los presos con el objetivo de asegurar los derechos humanos en un proceso plagado de vicios y elementos escabrosos, como el asesinato y la desaparición de detenidos. Además, desde el PCA se afirmaba que la acción contra el MTP estaba siendo utilizada para un reforzamiento del aparato represivo con el objetivo de restringir la democracia. Se trataba de una caracterización central porque ponía al descubierto que detrás del regreso de los "fantasmas antisubversivos" se escondía la intención gubernamental de contar con herramientas para enfrentar la agudización de la conflictividad social. En consonancia con esta postura, en aquellos meses, el PCA puso el foco de la crítica en el progresivo descalabro económico y en denunciar que la UCR, el PJ y la UCEDé representaban el mismo modelo de país.

Con relación a la presentación electoral, en 1988 el PCA participó de las elecciones internas del espacio "Izquierda Unida" (la que era entendida como la ampliación del FLNS), proponiendo candidatos en el marco del FRAL, en tanto el MAS presentó sus propios candidatos. Como resultado de esas elecciones internas quedó conformada una fórmula encabezada por Néstor Vicente, que obtuvo la candidatura presidencial, y Luis Zamora, del MAS, que quedó en segundo lugar.

En diciembre 1989 el Comité Central anunció la convocatoria al XVII Congreso en un clima de fuerte deliberación partidaria. La propuesta oficial postulaba el objetivo de ratificar, dar continuidad y profundizar la senda abierta por el "viraje" (Patricio Echegaray, 1989). Sin embargo, 1990 trajo novedades importantes que impactaron de lleno en la vida partidaria. En la escala internacional se produjo la caída del Muro de Berlín que venía a mostrar los signos inequívocos de la desintegración del bloque soviético. Ya durante el lustro anterior, el PCA había defendido de un modo complaciente la política de Perestroika, sin detenerse a reflexionar sobre el alcance de los cambios que introducía y el significado real del giro político. Pero, en 1990, esa realidad caía con tanta fuerza como los ladrillos del muro.

\footnotetext{
"Nuestro partido hoy vive de una manera particular la crisis de los proyectos socialistas; particularidad que surge de nuestra relación histórica no crítica con las experiencias y las ideas del llamado "socialismo real". Es una crisis que, teniendo un punto de concentración en la dirección nacional, se viene desarrollando de manera confusa y traumática, involucrando al conjunto del partido y de la FJC, agravando las dificultades de conducción, los problemas del accionar política y la concreción de un debate con parámetros claros, vinculados a la acción política, como único camino para la superación de nuestras limitaciones" (Proyecto de Tesis para el debate hacia el XVII Congreso, 1990: 58).
} 


\section{Natalia Casola}

En el plano nacional, la salida del gobierno de Alfonsín se produjo en medio de las luchas sociales desatadas por la hiperinflación, lo que reforzó la expectativa de una parte de la sociedad en torno del menemismo y de sus promesas de "revolución productiva". Al interior del partido, el "viraje", ese que había sido anhelado por la mayoría de la militancia, comenzó a mostrar sus imprecisiones. Durante ese año se libró un debate profundo que no escamoteó posiciones. Pese a ello, las Tesis del XVII Congreso ratificaron la línea definida en el congreso anterior.

La tesis central fue la defensa del FLNS concebido como la herramienta de unidad y "acumulación de fuerzas" para la "opción socialista". No obstante, y sin salir de los carriles de la continuidad con el "viraje", se formularon algunos balances críticos que introdujeron correcciones. Fundamentalmente, se admitió que el FLNS había sido concebido de un modo reduccionista, como sumatoria de fuerzas políticas, en lugar de bregar por transformarse en la herramienta de coordinación de múltiples fuerzas sociales, movimientos y organizaciones que no necesariamente se conformaban como partidos políticos o participaban de la contienda electoral. Se trataba de un análisis interesante porque daba cuenta del protagonismo que comenzaban a tener los movimientos sociales con base territorial, una presencia que preanunciaba la gestación del movimiento de desocupados. Sin embargo, como se dijo al inicio de este apartado, la reflexión en torno del FLNS desconsideraba, minimizaba o reducía el problema de la lucha de estrategias a meras contiendas de aparato por razones de sectarismo. El "pluralismo político e ideológico" que proponía el FLNS incorporaba programas y estrategias que bien podían convivir en el plano de las reivindicaciones inmediatas, incluso electorales, pero que difícilmente lograrían coexistir en una hipotética situación revolucionaria. Era esa potencial coyuntura la que quedaba sin resolver teóricamente. La revisión crítica de la idea de partido de vanguardia tal y como había concebida por Lenin, los llevó a proponer que la vanguardia unificada surgiría de la propia experiencia de lucha conjunta en el marco del FLNS, sin explicar qué papel cumpliría el PCA en todo ese proceso.

En consecuencia, la ratificación de la crítica al "etapismo" y la restitución del objetivo socialista reapareció despojado de dos conceptos que habían sido fundamentales en la tradición leninista: la construcción del partido de vanguardia, pensado como instrumento de dirección política, y el concepto de dictadura del proletariado. De este modo, el problema de la violencia revolucionaria quedó subsumido en una agenda de búsqueda de conciliación de los planteos de revolución, pluralidad y democracia. Con más preguntas que respuestas ingresaba el PCA a la última década del siglo XX.

\section{Conclusiones}

El propósito de este trabajo fue reconstruir la línea política del PCA durante la década de 1980 tomando como eje el análisis de la concepción en torno del Frente de Liberación Nacional y Social. A grandes rasgos, se ha demostrado que la evolución de la línea tuvo como momento bisagra el XVI Congreso partidario de 1986. Si hasta 


\section{EI Frente de Liberación Nacional y Social: en busca de una definición. EI PCA durante la posdictadura}

ese año la política frentista se mantuvo en los carriles del FDN, a partir de entonces, comenzó un proceso muy dinámico de progresiva e ininterrumpida radicalización. Sintéticamente, el cambio de contenido se materializó en un distanciamiento respecto de las tesis de construcción conjunta con el peronismo y en el acercamiento a las fuerzas políticas de izquierda. Esa voluntad se manifestó en el plano electoral con la constitución del Frente del Pueblo, y luego del FRAL y de la Izquierda Unida. Sin embargo, la política de FLNS dejó planteadas algunas indefiniciones. En primer lugar, el protagonismo del frentismo en desmedro del partido de vanguardia como herramienta de dirección, abrió el interrogante sobre el rol del PCA dentro del FLNS ¿Debía dirigirlo? ¿Acaso debía permanecer en él si prevalecía una estrategia contraria? ¿Cómo relacionarse con el resto de las fuerzas que integraban el FLNS? Hacia el final de la década, con las críticas ensayadas en el marco del XVII Congreso, las preguntas se multiplicaron. Si la premisa era constituir un frente junto con movimientos sociales y territoriales cuya pluralidad política se daba por descontada, cuál sería la utilidad de mantener la construcción de un partido de cuadros profesionales. Esas indefiniciones fueron acompañadas por otras. A pesar de la manifiesta intención de radicalizar sus posiciones, la desaparición del concepto de "dictadura del proletario" en los documentos del partido no fue reemplazada por ninguna otra noción, más allá de la voluntad explícita de combinar socialismo y democracia.

Así, en plena crisis del bloque soviético y del conjunto de la izquierda mundial, el PCA intentó abrirse camino y defender la senda abierta con el "viraje". La década siguiente, en pleno avance del neoliberalismo y con los discursos que anunciaban el final de las ideologías, puso a prueba cada una de sus proposiciones.

\section{Bibliografía}

Bona, V. (2019). La Ferifiesta como binóculo de la reconversión del Partido Comunista de la Argentina en la posdictadura: por la paz, la democracia y el socialismo. Ponencia presentada en XVII Jornadas Interescuelas de los Departamentos de Historia. UNC, Catamarca.

Browarnik, G. (2009). Sangre roja. Un estudio acerca de la transmisión de la tradición del Partido Comunista argentino durante la última dictadura militar y la posdictadura. Testimonios $\left(\mathrm{n}^{\circ} 1\right)$. Recuperado de https://hear.unr.edu.ar/wpcontent/uploads/2014/12/cortado.pdf

Camarero, H. (2014). "Tras las huellas de una ilusión: el Partido Comunista argentino y sus planteos del Frente Democrático Nacional (1955-1963)". En Archivos de Historia del Movimiento Obrero y la Izquierda, Año III, N5, pp. 31-50. 


\section{Natalia Casola}

Casola, N. (2015). El PC argentino y la dictadura militar. Militancia, estrategia política y represión estatal. Buenos Aires: Imago Mundi.

Casola, N. (2019). “Cuando se quebró el muro. Algunas notas acerca de la crisis en el Partido Comunista argentino durante los años 1980”. En Izquierdas, n49, pp.17521771. Recuperado en http://izquierdas.cl/ediciones/2020/numero-49

Echegaray, P. (1996). Utopía y liberación. Buenos Aires: Dirple.

Fernández Hellmund, P. (2015). Nicaragua debe sobrevivir. La solidaridad de la militancia comunista argentina con la Revolución Sandinista (1979-1990). Biuenos Aires: Imago Mundi.

Gilbert, I. (2009). La Fede. Buenos Aires: Sudamericana.

Nadra, A. (2012). Secretos en rojo, un militante entre dos siglos. Buenos Aires: Corregidor.

Schulman, J.E. (2008). Los laberintos de la memoria. Relatos de lucha contra la dictadura y la impunidad, Buenos Aires: El Folleto.

Sigal, J. (2006). El día que maté a mi padre, confesiones de un ex comunista. Buenos Aires: Sudamericana.

Staltari, S. (2014). "El Partido Comunista frente al peronismo: estrategia y tácticas políticas, 1945-1955”. En Archivos de Historia del Movimiento Obrero y la Izquierda, Año III, N5, pp. 11-30.

\section{Fuentes citadas}

Estatuto del Partido Comunista de la Argentina, Editorial Anteo, 1974

Echegaray, P. Opiniones de apertura expuestas por el camarada Patricio Echegaray para el debate en el Comité Central. Reunión del 8 de diciembre de 1989.

Fava A. Todos juntos por la liberación contra la dependencia. Intervención y resoluciones del XV Congreso, Buenos Aires, Editorial Anteo, 1983.

Fava A. Mensaje electoral al Partido y a la Juventud Comunista. Informe al Comité Central del Partido Comunista del 11 de septiembre de 1985, Buenos Aires, Editorial Anteo, Pp. 9 y 10. 


\section{EI Frente de Liberación Nacional y Social: en busca de una definición. El PCA durante la posdictadura}

Folleto, El resultado electoral y la unidad de la izquierda en torno al proyecto revolucionario. Reunión del Comité Central del Partido Comunista del 11 y 12 de septiembre de 1987.

Pereyra, J. "Construir una fuerza real y operativa en marcha hacia un partido comunista de masas". Informe rendido en la reunión del Comité Central del 24 y 30 agosto de 1984.

Proyecto de Tesis para el debate hacia el XVII Congreso. Aprobado por el CC del 25, 26 y 27 de julio de 1990.

Revista Nueva Era, n²12, diciembre de 1985.

Recibido: 07/08/2020

Evaluado: $15 / 09 / 2020$

Versión Final: 20/10/2020 